\title{
Dynamic monitoring of circulating tumour cells to evaluate therapeutic efficacy in advanced gastric cancer
}

\author{
Yilin Li ${ }^{1,2}$, Jifang Gong ${ }^{1,2}$, Qiyue Zhang ${ }^{1}$, Zhihao Lu ${ }^{1}$, Jing Gao ${ }^{1}$, Yanyan Li ${ }^{1}$, Yanshuo Cao ${ }^{1}$ and Lin Shen ${ }^{*, 1}$ \\ ${ }^{1}$ Key Laboratory of Carcinogenesis and Translational Research (Ministry of Education), Department of Gastrointestinal Oncology, \\ Peking University Cancer Hospital \& Institute, Beijing 100142, China
}

Background: This study was intended to determine the clinical significance of circulating tumour cells (CTCs) in patients with advanced gastric cancer (AGC), particularly the potential role of CTCs for dynamic monitoring of the therapeutic response.

Methods: A single-centre, prospective study was undertaken in 136 patients with newly diagnosed AGC. The patients' CTCs were enumerated using CellSearch at baseline and at the first response evaluation. In 15 patients whose clinical condition permitted longitudinal study, CTCs were longitudinally enumerated during treatment.

Results: Following 6 weeks of chemotherapy, an unfavourable post-therapy CTC level ( $\geqslant 3$ CTCs per $7.5 \mathrm{ml}$ ) was closely correlated with the objective response rate $(P=0.016)$ and the disease control rate $(P=0.013)$, and it also independently predicted a shorter progression-free survival and overall survival. Particularly, conversion to a favourable CTC level following therapy improved the prognosis, but patients who changed to an unfavourable CTC level fared significantly worse. Elevated CTCs during therapy may be associated with a poor prognosis.

Conclusions: Post-therapy CTC level may help in evaluating therapeutic response in patients with AGC and predicting their prognosis. In addition, changes in CTCs following therapy may be useful in rapidly identifying ineffective treatments and poor prognosis.

Gastric cancer (GC) remains one of the most common causes of cancer deaths worldwide (Jemal et al, 2011). Despite improvements in cancer diagnosis and therapy, about two-thirds of patients with GC are still diagnosed with advanced gastric cancer (AGC) in many developing countries, including China (Shen et al, 2013). Chemotherapy or targeted therapy remain mainstays of treatment for AGC (Hartgrink et al, 2009; Shen et al, 2013). Unfortunately, diminished sensitivity to chemotherapy and the invariable development of acquired resistance limit the efficacy of treatment. In general, $<60 \%$ of patients with AGC have an effective response to chemotherapy, which results in overall 5-year survival rates as low as 20\% (Hartgrink et al, 2009; Shen et al, 2013). Identification of tumour tissue-based biomarkers related to therapeutic responsiveness may help screen sensitive patients before therapy (Scartozzi et al, 2009; Gao et al, 2011; Lu et al, 2011; Liang and
Kim, 2013; Wang et al, 2014). However, as it is challenging to obtain tumour tissues repeatedly in AGC patients, tumour tissuebased biomarkers may be inappropriate to serve as continuous real-time monitors of acquired resistance during clinical therapy (Alix-Panabières and Pantel, 2013; Haber and Velculescu, 2014).

Circulating tumour cells (CTCs) in peripheral blood are an attractive alternative that might provide the ability to repeatedly and non-invasively monitor therapeutic responses in cancer patients (Yu et al, 2011; Alix-Panabières and Pantel, 2013; Balic et al, 2013; Haber and Velculescu, 2014). A number of studies have reported that enumeration of CTCs using CellSearch (Veridex, Raitan, NJ, USA) can be used for predicting chemotherapy effectiveness in various cancers, including metastatic breast cancer (Cristofanilli et al, 2004, 2005; Mostert et al, 2009; Castle et al, 2014), castration-resistant prostate cancer (de Bono et al, 2008;

*Correspondence: Professor L Shen; E-mail: linshenpku@163.com

${ }^{2}$ These authors contributed equally to this work.

Received 14 August 2015; revised 8 October 2015; accepted 26 October 2015

(c) 2016 Cancer Research UK. All rights reserved 0007-0920/16 
Scher et al, 2009; Miyamoto et al, 2014), metastatic colorectal cancer (Cohen, 2009; Cohen et al, 2009; Rahbari et al, 2010; Lim et al, 2014), non-small cell lung cancer (Krebs et al, 2011; Wang et al, 2013; Alama et al, 2014), and small cell lung cancer (Hou et al, 2009, 2012; Hiltermann et al, 2012). Continuous enumeration of CTCs in these cancers has been successfully achieved, and the findings of a number of studies suggest that patients with persistently unfavourable CTC level and those who convert to an unfavourable CTC level post therapy may exhibit limited sensitivity to current treatments (Cristofanilli et al, 2004, 2005; Danila et al, 2007; de Bono et al, 2008; Cohen, 2009). Consequently, real-time monitoring of the CTC level might help in rapidly identifying therapeutic resistance and might allow the selection of effective therapies without waiting for radiographic evidence of response or non-response.

In patients with AGC, clinical evidence of the value of CTCs in predicting and monitoring therapeutic responses remains limited (Tang et al, 2013; Tsujiura et al, 2014). A study of 52 Japanese AGC patients suggested that an unfavourable CTC level at 2 or 4 weeks after beginning chemotherapy may be correlated with an inferior therapeutic outcome, as patients with unfavourable CTC level at these time points had shorter median progression-free survival (mPFS) and overall survival (mOS) in comparison with patients with favourable CTC levels (Matsusaka et al, 2010). However, more robust data based on studies of longer duration are needed to confirm the predictive ability of CTCs in AGC. Importantly, as noted above, changes in CTCs in response to therapy need to be studied further to confirm their value in realtime monitoring of therapeutic efficacy and their capacity to rapidly identify therapeutic resistance during treatment.

To investigate the clinical significance of CTCs in AGC, we undertook a single-centre, prospective study of Chinese patients with newly diagnosed AGC (ClinicalTrials.gov identifier NCT01625702). The correlation of changes in CTC levels with the patients' clinical prognoses and the value of serial changes of CTC levels in individual patients during therapy were investigated to evaluate if CTCs can be used to monitor the response to AGC therapies.

\section{MATERIALS AND METHODS}

Patients and sample collection. A total of 138 patients with newly diagnosed AGC were prospectively enrolled in the study at the Peking University Cancer Hospital between July 2012 and July 2014. The patients were $>18$ years of age and had histologically confirmed, locally advanced or recurrent and/or metastatic adenocarcinoma of the stomach or gastroesophageal junction. Patients who had received no prior treatment for advanced/ metastatic disease and who had Karnofsky performance status $\geqslant 60$, adequate organ function, and measurable or non-measurable but evaluable disease were eligible for the study. The enrolled patients were mainly treated with first-line paclitaxel (PTX) or platinum (DDP)-based chemotherapy, the regimens for which were similar to those used by us in a previous study (Gao et al, 2011). After 6 weeks of treatment (two cycles of chemotherapy), evaluation of the clinical response was performed by computed tomography according to RECIST version 1.1 criteria (Eisenhauer et al, 2009). For GC, the primary tumour has been considered unmeasurable according to RECIST. Therefore, uni-dimensional measurement of lymph nodes or distant metastases is suggested in assessing response using clinical imaging. Responses were categorised as partial responses (PR, i.e., at least a 30\% decrease in the sum of diameters of target lesions), progressive disease (PD, i.e., at least a $20 \%$ increase in the sum of diameters of target lesions), or stable disease (SD, neither sufficient shrinkage to qualify as a PR nor a sufficient increase to qualify as PD) (Eisenhauer et al, 2009).

Peripheral blood samples of $7.5 \mathrm{ml}$ were collected from the patients for CTC enumeration. The blood samples were drawn before therapy (baseline) and at the time of the first response evaluation (after 6 weeks of therapy). In 15 patients whose clinical condition permitted, serial blood samples were collected at intervals of 6 weeks.

Consent forms were provided in writing to all patients to inform them that their blood samples were to be used for future research. The study was approved by the Ethics Committee of Peking University Cancer Hospital and was performed according to the principles of the Declaration of Helsinki.

CTC enumeration. CTCs were enumerated by the CellSearch system as described previously (Matsusaka et al, 2010). In brief, $7.5 \mathrm{ml}$ blood samples were drawn into $10 \mathrm{ml}$ CellSave Vacutainer tubes (Becton Dickinson, Franklin Lakes, NJ, USA) containing ethylenediaminetetraacetic acid and cell fixative. Samples were kept at room temperature for up to $72 \mathrm{~h}$, followed by processing with a semi-automated system (CellPrep) and a CellSearch Epithelial Cell Kit. Cells expressing epithelial cell adhesion molecule were captured with antibody-coated ferrous particles, and cell nuclei were labelled with $4^{\prime}$,6-diamidino-2-phenylindole. Fluorescent dye-labelled monoclonal antibodies against leukocytes (CD45allophycocyan) and cytokeratins (CK8, 18, 19-phycoerythrin) were used to distinguish epithelial cells from leukocytes. Further identification and enumeration of CTCs were performed using the CellSpotter Analyzer, a semi-automated fluorescence-based microscopy system that permits computer-generated reconstruction of cellular images. CTCs were defined as nucleated cells with CK-positive and CD45-negative staining.

Statistical analyses. The primary end point of the study was OS. Based on an unfavourable threshold level of $\geqslant 3$ CTCs per $7.5 \mathrm{ml}$ of blood, it was assumed that the ratio of patients in the favourable and unfavourable CTC groups would be $4: 1$ and that the study would last for 29 months, with patient accrual (entry) occurring in the first 18 months and $10 \%$ of patients dropping out during the study. Based on these assumptions, a minimum of 102 patients, 20 in the unfavourable CTC group and 82 in the favourable CTC group, was the number calculated to achieve $80 \%$ power for detecting an improvement of 8.5 months in the mOS in the favourable CTC group compared with a mOS of 7.5 months in unfavourable CTC group (two-sided log-rank $P$-value of 0.1 ).

Statistical analysis was performed using SPSS 18.0 software (SPSS Inc., Chicago, IL, USA). The correlations of CTC numbers with clinicopathologic characteristics and clinical responses were assessed using, respectively, Pearson $\chi^{2}$-test and Fisher's exact test. The threshold of CTCs established was based on nonparametric receiver operating characteristic (ROC) analysis, and optimal cutoff values were determined to maximise the Youden index. The Youden index (sensitivity + specificity -1 ) is determined by selecting a point that maximises the number of subjects who are classified correctly and gives equal weight to sensitivity and specificity (Youden, 1950). PFS was defined as the time from initial blood collection to the date clinical progression was confirmed or was censored at the last follow-up. OS was defined as the time from initial blood collection to the date death occurred or was censored at the last follow-up. Kaplan-Meier survival plots for PFS or OS were generated based on CTC levels before and after therapy, and the survival curves were compared using log-rank tests. Cox proportional hazards regression was used to determine the univariate and multivariate hazard ratios of PFS and OS. Baseline and post-therapeutic CTC numbers and standard clinical factors including liver and peritoneal metastases, the Lauren classification, human epidermal growth factor receptor 2 (HER2) status, and the 
type of treatment were subjected to univariate analysis for both PFS and OS. Significant parameters in the univariate analysis were then included in the multivariate analysis. A two-sided $P$-value $<0.05$ was considered statistically significant.

\section{RESULTS}

Patient characteristics. Of the 138 Chinese patients with AGC who were prospectively enrolled in the study between July 2012 and January 2014, 136 met the study's inclusion/exclusion criteria. The flow of patients in the study is summarised in Supplementary Figure S1, and the clinicopathological characteristics of the 136 evaluable patients are shown in Supplementary Table S1. At the time the clinical response was analysed, 106 patients (78.0\%) were assessable, of whom 85 (80.2\%) had progressive disease; $75(70.8 \%)$ of these patients died. The median follow-up time was 28.3 months, and the mPFS and mOS were 6.5 months (95\% CI 5.1-8.0 months) and 14.0 months (95\% CI 11.8-16.2 months), respectively.

CTC numbers at baseline and their correlation with clinicopathological characteristics. At baseline, $\geqslant 1$ CTC (per $7.5 \mathrm{ml}$ ) could be detected in $55.9 \%$ of patients. The median number of CTCs detected at baseline was 1 (range, 0-1191) and the mean number ( \pm s.d.) was $42( \pm 144)$. After 6 weeks of therapy, the median and mean number of CTCs were decreased to 0 (range $0-600)$ and $12( \pm 64)$ (Supplementary Figure S2), respectively. The correlation between CTC numbers and clinicopathological characteristics is shown in Table 1 . CTC numbers $\geqslant 3, \geqslant 4$, and $\geqslant 5$ per $7.5 \mathrm{ml}$ were detected significantly more frequently in patients with primary tumours in the stomach than in those with primary tumours in the gastroesophageal junction. In addition, the proportion of patients with $\geqslant 1, \geqslant 3, \geqslant 4$, and $\geqslant 5$ CTCs per
$7.5 \mathrm{ml}$ was significantly higher in patients with liver metastases than in those without liver metastases. Other clinicopathological characteristics such as the Lauren classification, peritoneal metastases, and HER2 status were not significantly correlated with CTC numbers.

Establishing the optimal threshold for CTCs. To establish the optimal threshold of CTCs that most clearly predicts therapeutic responsiveness, PFS and OS, ROC curves, and Youden indexes were estimated for both baseline and post-therapy CTCs predicting OS (Supplementary Figure S3A and B) (Youden, 1950). As shown in Supplementary Figure S3B, the cutoff points of 1-3 for baseline CTCs and 1-5 for post-therapy CTCs all exhibited larger Youden indexes. When the areas under the ROC curves (AUC) for CTC thresholds of 1-5 were compared, a cutoff value of 3 for both baseline and post-therapy CTCs maximised the AUC area $(0.583$ for baseline CTCs; 0.648 for post-therapy CTCs) (Supplementary Figure S3C and D). Therefore, the cutoff of $\geqslant 3$ CTCs per $7.5 \mathrm{ml}$ was selected as the unfavourable threshold for further investigations.

Correlation between CTC numbers and therapeutic response as assessed by imaging. At the first response evaluation, $\mathrm{PR}, \mathrm{SD}$, and PD were achieved in 26 (24.5\%), 70 (66.0\%), and $10(9.4 \%)$ patients, respectively. The overall response rate (ORR) and disease control rate (DCR) were $24.5 \%$ and $90.6 \%$, respectively. As shown in Table 2, the ORR and DCR were not significantly different in patients with unfavourable and favourable numbers of CTCs at baseline $(24.4 \%$ and $86.7 \%$, respectively, vs $24.6 \%$ and $93.4 \%$, respectively). However, following 6 weeks of therapy, the ORR and DCR in the group with unfavourable post-therapy CTC numbers were only $7.7 \%$ and $76.9 \%$, respectively, which were significantly lower than those observed in the group with favourable posttherapy CTC numbers $(30.0 \%$ and $95.0 \%$, respectively; $P=0.016$

Table 1. Prevalence of CTCs at baseline and their association with clinical characteristics $(n=136)$

\begin{tabular}{|c|c|c|c|c|c|c|}
\hline & & \multicolumn{5}{|c|}{ CTC numbers (per $7.5 \mathrm{ml}$ ) ( $\mathrm{n} \%$ of patients) } \\
\hline All AGCs & 136 & $76(55.9)$ & $65(47.8)$ & $57(41.9)$ & $52(38.2)$ & $48(35.3)$ \\
\hline Stomach & 85 & $49(57.6)$ & $45(52.9)$ & $42(49.4)$ & $39(45.9)$ & $37(43.5)$ \\
\hline $\begin{array}{l}\text { Gastroesophageal junction } \\
(P \text {-value })^{\mathbf{b}}\end{array}$ & 51 & $\begin{array}{c}27(52.9) \\
(0.593)\end{array}$ & $\begin{array}{c}20(39.2) \\
(0.121)\end{array}$ & $\begin{array}{l}15(29.4) \\
(0.022)\end{array}$ & $\begin{array}{l}13(25.5) \\
(0.018)\end{array}$ & $\begin{array}{l}11(21.6) \\
(0.009)\end{array}$ \\
\hline Diffuse & 42 & $24(57.1)$ & $22(52.4)$ & $20(47.6)$ & $17(40.5)$ & $15(35.7)$ \\
\hline Mixed & 32 & $20(62.5)$ & $16(50.0)$ & $13(40.6)$ & $13(40.6)$ & $13(40.6)$ \\
\hline$(P$-value) & & $(0.556)$ & $(0.682)$ & $(0.657)$ & $(0.816)$ & $0.689)$ \\
\hline \multicolumn{7}{|l|}{ Sites of metastasis } \\
\hline \multicolumn{7}{|l|}{ Liver } \\
\hline Yes & 36 & $25(69.4)$ & $20(55.6)$ & $20(55.6)$ & $20(55.6)$ & $18(50.0)$ \\
\hline No & 112 & $61(54.5)$ & $53(47.3)$ & $46(40.9)$ & $46(41.1)$ & $44(39.3)$ \\
\hline$(P$-value $)$ & & $(0.313)$ & $(0.494)$ & $(0.605)$ & $(0.417)$ & $(0.382)$ \\
\hline \multicolumn{7}{|l|}{ HER2 status ${ }^{d}$} \\
\hline Positive & 29 & $17(58.6)$ & $16(55.2)$ & $14(48.3)$ & $14(48.3)$ & $12(41.4)$ \\
\hline Negative & 101 & $55(54.5)$ & $45(44.6)$ & $40(39.6)$ & $36(35.6)$ & $34(33.7)$ \\
\hline (P-value) & & $(0.691)$ & $(0.313)$ & $(0.404)$ & $(0.218)$ & $(0.444)$ \\
\hline \multirow{2}{*}{\multicolumn{7}{|c|}{ Abbreviations: AGC = advanced gastric cancer; HER2 = human epidermal growth factor receptor 2.}} \\
\hline & & & & & & \\
\hline \multicolumn{7}{|c|}{ 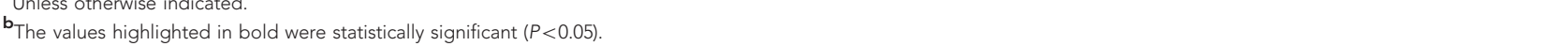 } \\
\hline \multicolumn{7}{|c|}{${ }^{{ }^{c} F i v e ~ p a t i e n t s ~ h a d ~ u n a v a i l a b l e ~ L a u r e n ~ c l a s s i f i c a t i o n s . ~}$} \\
\hline${ }^{d}$ Six patients had unavailable $\mathrm{HE}$ & status information. & & & & & \\
\hline
\end{tabular}


and $P=0.013)$. These results indicate that an unfavourable post-therapy CTC level was closely correlated with a poor therapeutic response.

The use of CTC numbers to predict PFS and OS. Kaplan-Meier plots for predicting PFS and OS with the baseline CTC numbers are shown in Figure $1 \mathrm{~A}$ and $\mathrm{B}$. The mPFS of patients with unfavourable baseline CTCs was 5.7 months (95\% CI 4.0-7.3 months). Although the mPFS was relatively shorter, it was still not significantly different from that of patients with favourable baseline CTCs (7.1 months, 95\% CI 5.6-8.7 months, $P=0.095$ ) (Figure 1A). However, the mOS of patients with unfavourable baseline CTCs (11.9 months, 95\% CI 8.9-14.9 months) was significantly shorter than that of patients with favourable baseline CTCs (17.7 months, 95\% CI 14.1-21.3 months; $P=0.004$ ) (Figure 1B). Further analysis of the PFS and OS in patients receiving different types of chemotherapy revealed that the predictive value of CTCs may be more significant in patients receiving DDP-based

\begin{tabular}{l} 
Table 2. CTCs and their correlation with response ( $n=106)$ \\
\cline { 3 - 6 }
\end{tabular}
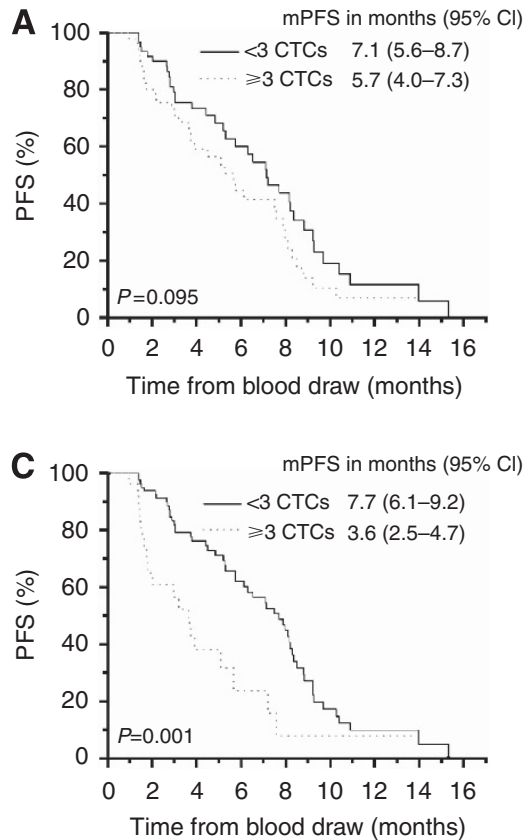

therapy in comparison with those receiving PTX-based therapy (Supplementary Figure S4).

After 6 weeks of therapy, the mPFS of patients who still had unfavourable CTC numbers was 3.6 months (95\% CI 2.5-4.7 months), which was significantly shorter than that observed in those with favourable post-therapy CTC numbers (7.7 months, 95\% CI 6.1-9.2 months; $P=0.001$ ) (Figure 1C). Similarly, patients with unfavourable post-therapy CTC numbers had an inferior mOS (8.0 months, 95\% CI 2.3-13.7 months) in comparison with those with favourable post-therapy CTC numbers (17.7 months, 95\% CI 14.6-20.8 months; $P<0.001$ (Figure 1D). Thus, unfavourable CTC levels, especially after therapy, may help predict an inferior PFS and OS.

To evaluate the independent predictive effect of CTCs for PFS and OS, univariate and multivariate Cox proportional hazards regression were performed. The results shown in Supplementary Table S2 for the univariate analysis indicate that baseline CTCs, peritoneal metastasis, HER2 status, and treatments were significantly associated with OS, whereas the post-therapy CTCs and the Lauren classification were significantly associated with both OS and PFS. However, the multivariate analysis (Supplementary Table S2) showed that only the post-therapy CTCs and the Lauren classification remained as predictors of PFS and OS. Thus, after adjusting for clinically significant factors, the post-therapy CTC number remained an independent predictor of PFS (HR 2.152, 95\% CI 1.114-4.156; $P=0.022$ ) and OS (HR 3.463, 95\% CI 1.822-6.584; $P<0.001)$ in patients with AGC.

Therapeutic assessments using combined post-therapy CTCs and imaging. In view of the close correlation of post-therapy CTCs with therapeutic response, PFS, and OS, it is reasonable to deduce that therapeutic effectiveness and prognosis can be more precisely evaluated using a combination of post-therapy CTCs and imaging. As shown in Figure 2A, the OS for patients with PR or SD assessed by imaging was significantly longer than that of patients with PD. Combination with CTC enumeration revealed that the OS of patients with favourable post-therapy CTC numbers and non-PD (PR or SD, Group 1) was significantly longer than that of patients with favourable post-therapy CTC numbers and PD (Group 2), unfavourable post-therapy CTC numbers and
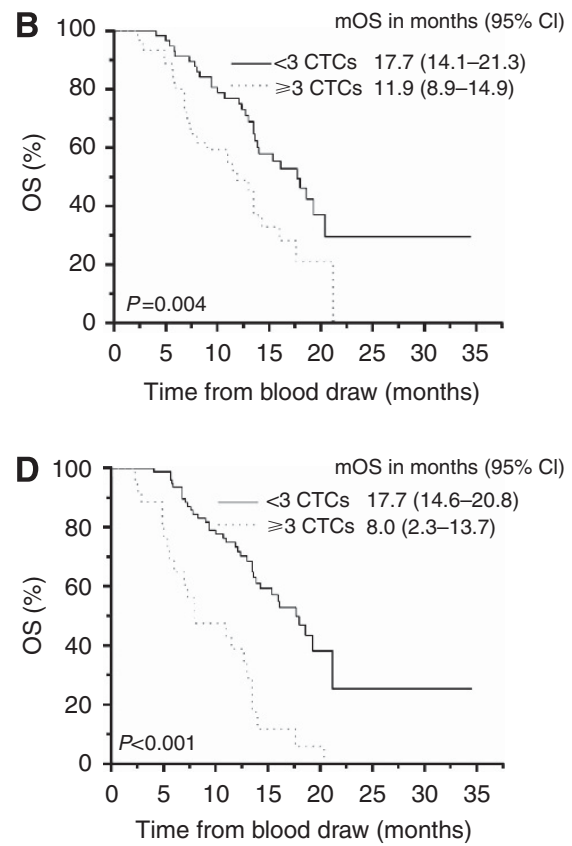

Figure 1. Kaplan-Meier curves for PFS (A and $\mathbf{C}$ ) and OS (B and $\mathbf{D})$ in patients with favourable and unfavourable CTC numbers at baseline (A and $\mathbf{B})$ or post therapy (C and $\mathbf{D})$. 

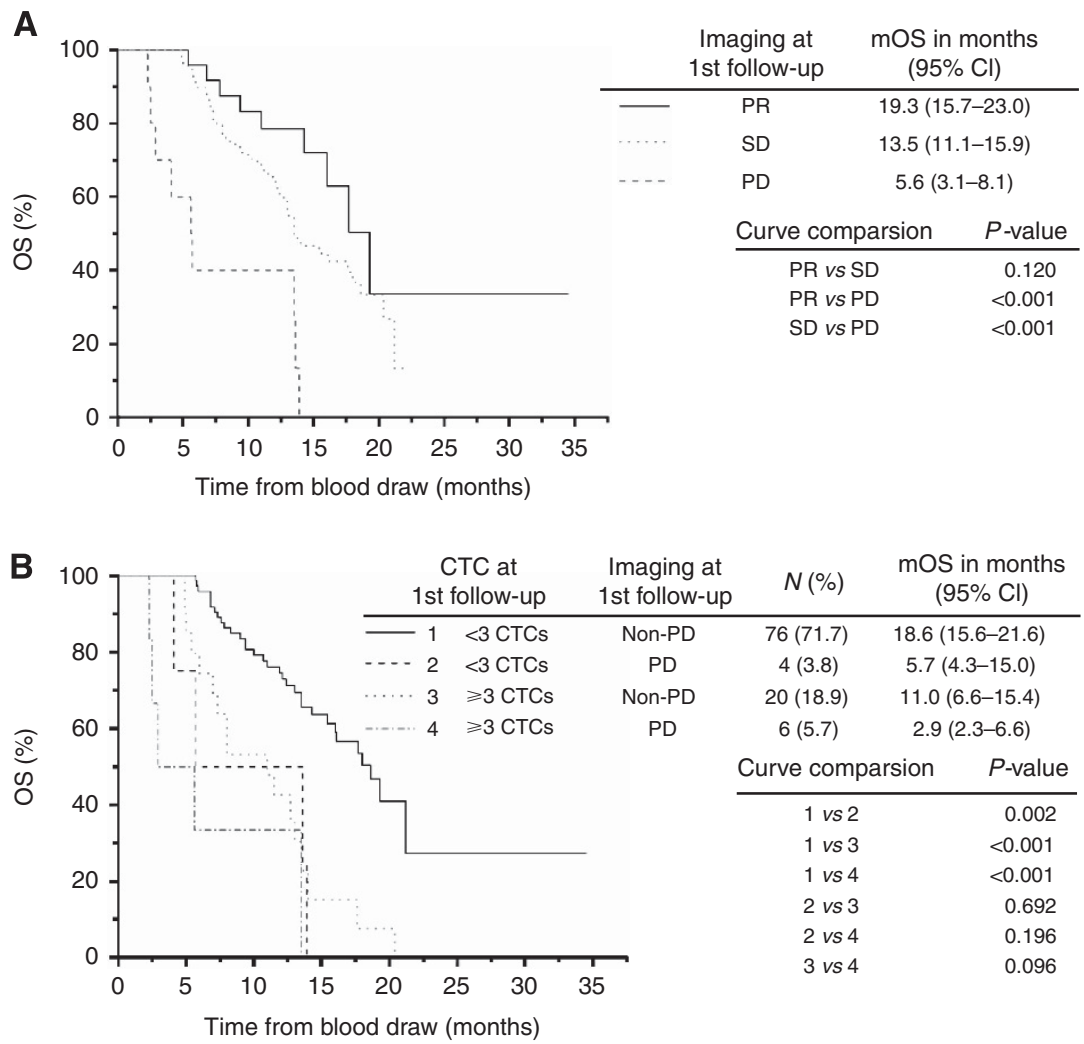

Figure 2. Relationship of imaging and CTCs to OS. (A) Kaplan-Meier curves for OS in patients who achieved PR, SD or PD responses as assessed by imaging. (B) Kaplan-Meier curves for OS based on a combination of CTC enumeration and imaging. The first follow-up indicates the time point following 6 weeks of chemotherapy. PD = progressive disease; non-PD includes PR or SD; PR = partial response; $S D=$ stable disease.

non-PD (Group 3), and unfavourable post-therapy CTC numbers and $\mathrm{PD}$ (Group 4) (Figure 2B). These results suggest that either elevated CTCs or PD as assessed by imaging indicate ineffective therapy and poor prognosis. Thus, CTCs in combination with imaging may potentially be used to predict therapeutic end points and need for treatment changes.

CTC changes in response to therapy. To further assess the benefit of CTCs, serial changes in CTCs in response to therapy were studied. Based on the change in CTC numbers from baseline to the time of the first response evaluation, the assessable patients were subdivided into four groups: Group 1, patients having persistently favourable CTC numbers; Group 2, patients having favourable CTC numbers at baseline who converted to unfavourable CTC numbers post therapy; Group 3, patients having unfavourable CTC numbers at baseline who converted to favourable CTC numbers post therapy; and Group 4, patients having persistently unfavourable CTC numbers. As shown in Figure 3, patients in Groups 1 and 3 had almost equal PFS and OS benefits, which was also observed in Group 2 and 4. However, the mPFS of Groups 1 and 3 (7.7 and 7.9 months, respectively) was considerably longer than that of Groups 2 and 4 (3.2 and 3.6 months, respectively). Similarly, the mOS of Groups 1 and 3 (18.6 and 14.3 months, respectively) was also longer than that of Group 4 ( 8.0 months). These findings indicate that patients with persistently favourable CTCs or those exhibiting an early conversion to favourable CTC numbers following therapy may have an improved PFS and OS. In contrast, patients who have persistently unfavourable CTCs or convert from favourable CTC numbers at baseline to unfavourable CTC numbers post therapy have an inferior PFS and OS.

Longitudinal changes of CTCs during therapy were monitored in real-time in 15 patients. As shown in Figure 4, not all CTC changes
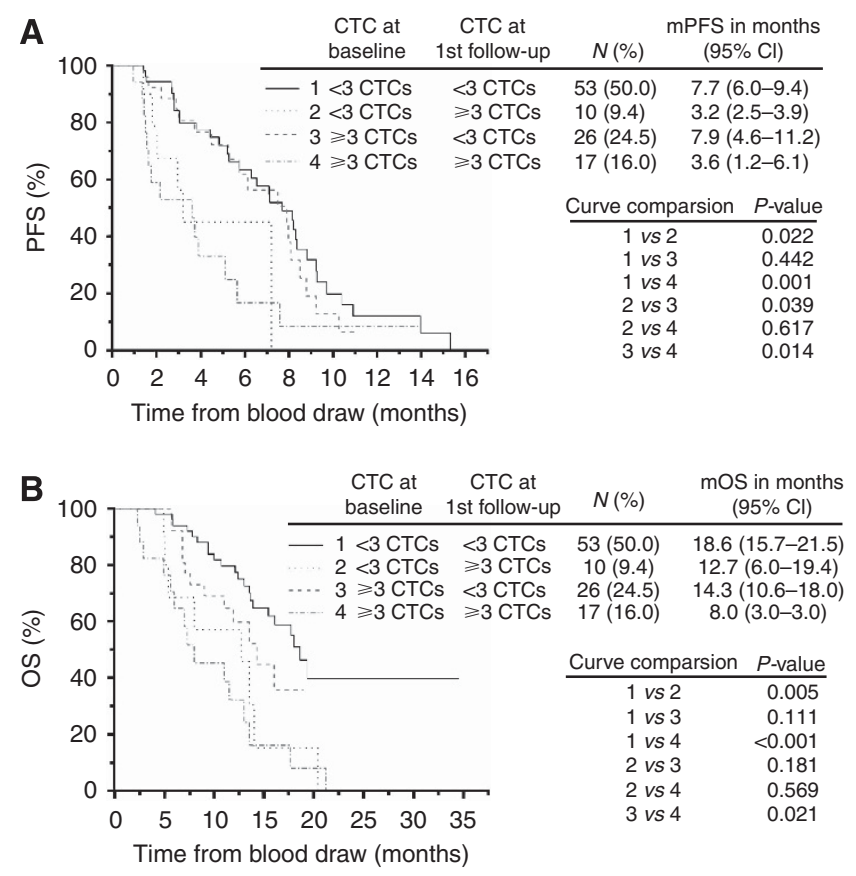

Figure 3. Correlation of changes in CTCs following therapy with PFS (A) and OS (B). The patients were divided into four groups according to their changes in CTCs from baseline to post therapy. Group 1: $<3$ CTCs at baseline and post therapy; Group 2: $<3$ CTCs at baseline and $\geqslant 3$ CTCs post therapy; Group 3: $\geqslant 3$ CTCs at baseline and $<3$ CTCs post therapy; Group 4: $\geqslant 3$ CTCs at baseline and post therapy. The first follow-up indicates the time point following 6 weeks of chemotherapy. 

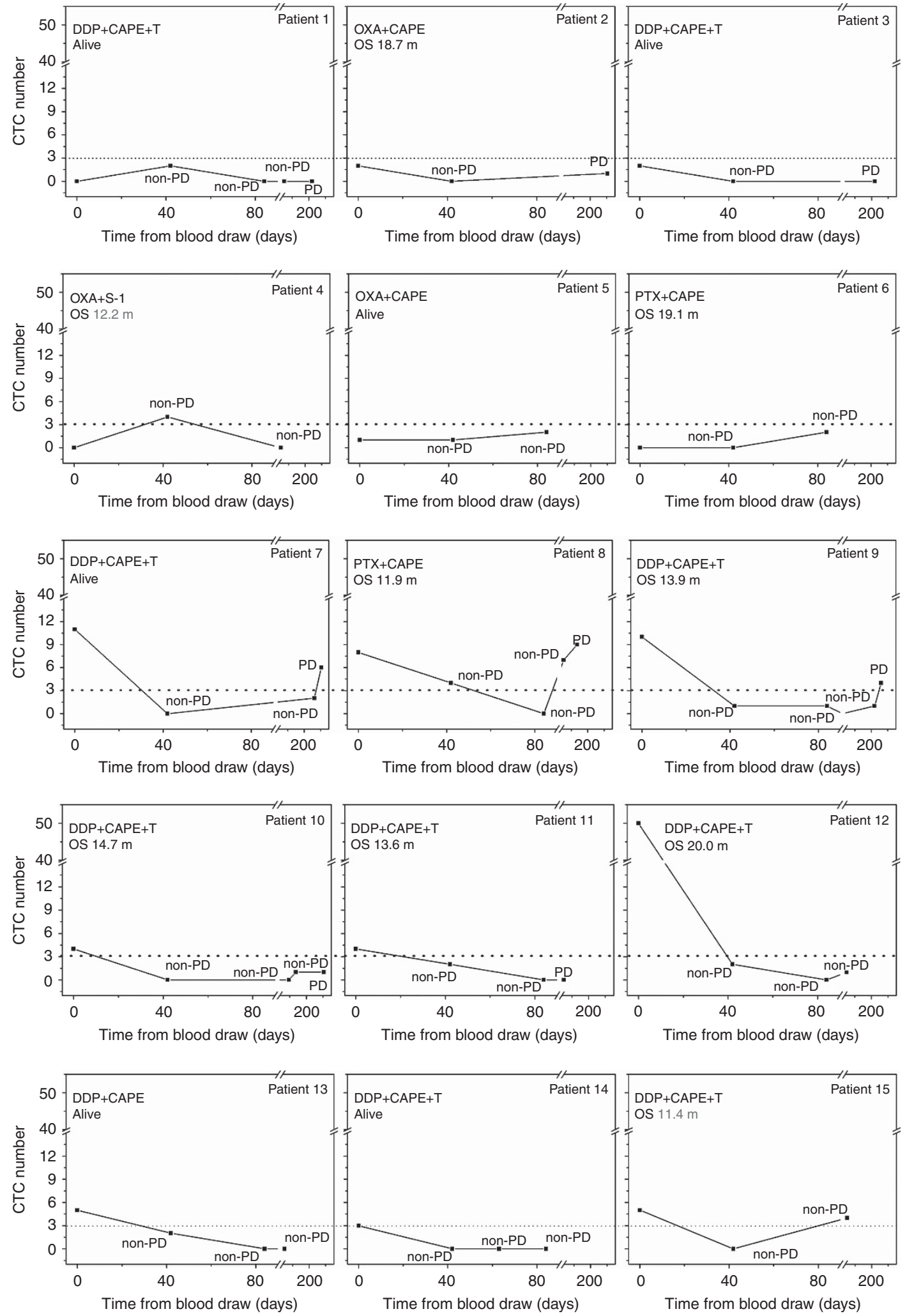

Figure 4. Longitudinal monitoring of CTC changes in response to therapy in patients 1-15. Patients 1, 3, 5, 7, 13, and 14 were still alive at the time of analysis. DDP = cisplatin; $\mathrm{CAPE}=$ capecitabine; $\mathrm{T}=$ trastuzumab; OXA = oxaliplatin; $\mathrm{PTX}=$ paclitaxel.

were exactly correlated to the imaging results. Thirteen of the 15 assessable patients exhibited favourable CTC numbers when they had an effective response to therapy (non-PD). In patients 4 and 15, the CTC numbers were elevated when they are still non-PD. However, the OS of these two patients of 12.2 and 11.4 months, respectively, were relatively shorter than that of other patients. As such, it seems that the elevated CTCs may indicate the poor prognosis. Further, when resistance to therapy occurred (PD), CTC levels were not consistently elevated in all patients. In patients with favourable baseline CTC numbers (patients 1-6 in Figure 4), none exhibited elevated CTCs when PD occurred. However, among five patients with unfavourable baseline CTCs who had PD during the 
study (patient 7-11 in Figure 4), three of them (60\%) (patients 7, 8, and 9) exhibited unfavourable CTC numbers at the time of disease progression. These results suggested that the dynamic changes of CTCs may need to be monitored and the elevated CTCs may correspond with an ineffective therapeutic response, especially in patients with unfavourable baseline CTC numbers.

\section{DISCUSSION}

Although evidence of the effectiveness of CTCs for predicting therapeutic responsiveness has been reported for various cancers (Yu et al, 2011; Alix-Panabières and Pantel, 2013; Balic et al, 2013; Haber and Velculescu, 2014), the evidence for AGC is currently limited (Tang et al, 2013; Tsujiura et al, 2014). In this study, the baseline CTC number was not significantly correlated with the therapeutic response or PFS. However, patients with unfavourable baseline CTC numbers did have a significantly shorter mOS than patients with favourable CTC numbers. Following 6 weeks of chemotherapy, unfavourable post-therapy CTC levels were significantly correlated with an ineffective therapeutic response, and these numbers independently predicted a decreased PFS and OS. Although the results suggesting the predictive value of baseline CTCs in patients with AGC need to be further verified in larger sample-size studies, post-therapy CTCs clearly help in predicting therapeutic responsiveness and the prognosis. Furthermore, a combination of post-therapy CTC enumeration and imaging may help to more precisely assess therapeutic responsiveness, as either unfavourable post-therapy CTC numbers or PD may indicate ineffective therapy and a poor prognosis.

Although a one-time assessment of CTCs may be important in screening patients for therapeutic responsiveness, the change in CTCs in an individual patient following a therapeutic intervention appears most likely to be of significant clinical value. Therefore, dynamic changes in CTCs in response to therapy were investigated in the present study. As with previously reported, findings in patients with metastatic breast cancer (Cristofanilli et al, 2004, 2005), castration-resistant prostate cancer (Danila et al, 2007; de Bono et al, 2008), and metastatic colorectal cancer (Cohen, 2009), AGC patients exhibiting an early conversion to favourable CTC numbers after therapy appeared to have an improved PFS and OS. In contrast, patients with persistently unfavourable CTCs or conversion to unfavourable CTC numbers post therapy exhibited an inferior PFS and OS, indicating a limited response to therapy. Monitoring of dynamic changes in CTCs during therapy in 15 AGC patients in our study indicated that CTC changes in patients with unfavourable baseline CTC numbers seems to be more correlated to the imaging results, and an increased CTC level following therapy in individual patients may indicate potential resistance to treatment. Consequently, CTCs may be a useful alternative to imaging for assessing treatment resistance, which underlines their potential in guiding treatment selection.

To confirm this hypothesis, additional studies of the significance of CTCs in guiding changes in the treatment of AGC patients should be designed. For example, it needs to be clarified whether persistently unfavourable CTCs or a conversion to unfavourable CTCs after therapy in AGC patients predicts therapy end points and whether a switch to a new line of treatment can contribute to an improved PFS or OS. In several recent randomised, phase III clinical trials in breast cancer, evaluation of CTC changes were incorporated into the study design to explore their predictive value in guiding treatment selections (Castle et al, 2014). Similarly, in a study of castration-resistant prostate cancer, CTCs were explored as interim or potential surrogate markers for clinical end points, with the hypothesis that an elevation of CTCs following clinical treatment may be an early sign of therapeutic resistance and a true indicator of the need for a treatment change (Miyamoto et al, 2014).

In addition to CTC enumeration, identification of the CTC subtype may help in diagnosing and selecting patients for treatment, especially for targeted therapies. The molecular characteristics of CTCs can also be expected to help in the discovery of novel therapeutic agents and to facilitate their selection in individual patients (Theodoropoulos et al, 2010; Yu et al, 2011; Liang and Kim, 2013; Haber and Velculescu, 2014). Indeed, our previous study of the phenotypic and karyotypic characterisation of CTCs in AGC has suggested that the HER2 status of CTCs may help in predicting the therapeutic efficacy of anti-HER2 targeted therapy (Li et al, 2014). In addition, different ploidies of chromosome 8 in the CTCs of patients with AGC are closely correlated with the sensitivity of and resistance to PTX or DDP-based chemotherapy (Li et al, 2014). Thus, multiplex molecular analysis of CTCs, via methods such as gene and protein profiling, should further improve current therapeutic strategies for AGC.

In conclusion, the findings of this study indicate that unfavourable post-therapy CTC levels are closely associated with a poor therapeutic outcome and are an independent predictor of an inferior PFS and OS in Chinese patients with AGC. Moreover, a combination of post-therapy CTC enumeration and imaging was found to be a precise method for screening patients for therapeutic responsiveness. Importantly, a persistently favourable CTC level or an early conversion to a favourable CTC level following therapy was found to indicate an improved prognosis. However, patients with a persistently unfavourable CTC level or those who converted to an unfavourable CTC level following therapy fared significantly worse, implying a limited response to the treatment administered. Longitudinal changes of CTCs in response to therapy may help in rapidly indicating acquired resistance in individual patients before they are radiographically evaluated. These results confirm the clinical significance of CTCs for therapy predictions. Further studies are ongoing to assess whether evaluation of the changes in CTC numbers and the molecular profiling of CTCs are beneficial for selecting treatment in patients with AGC.

\section{ACKNOWLEDGEMENTS}

This study was supported by NSFC projects $(81301323,81472789)$, the National High Technology Research and Development Program of China (No. 2012AA 02A 504) and the National Basic Research Program of China (No. 2014CBA02002).

\section{CONFLICT OF INTEREST}

The authors declare no conflict of interest.

\section{REFERENCES}

Alama A, Truini A, Coco S, Genova C, Grossi F (2014) Prognostic and predictive relevance of circulating tumor cells in patients with non-smallcell lung cancer. Drug Discov Today 19(10): 1671-1676.

Alix-Panabières C, Pantel K (2013) Circulating tumor cells: liquid biopsy of cancer. Clin Chem 59(1): 110-118.

Balic M, Williams A, Lin H, Datar R, Cote RJ (2013) Circulating tumor cells: from bench to bedside. Annu Rev Med 64: 31-44.

Castle J, Shaker H, Morris K, Tugwood JD, Kirwan CC (2014) The significance of circulating tumour cells in breast cancer: a review. Breast 23(5): 552-560.

Cohen SJ (2009) Relationship of circulating tumor cells to tumor response, progression-sree durvival, and overall survival in patients with metastatic colorectal cancer. J Clin Oncol 27(11): 1923-1923. 
Cohen SJ, Punt CJ, Iannotti N, Saidman BH, Sabbath KD, Gabrail NY, Picus J, Morse MA, Mitchell E, Miller MC, Doyle GV, Tissing H, Terstappen LW, Meropol NJ (2009) Prognostic significance of circulating tumor cells in patients with metastatic colorectal cancer. Ann Oncol 20(7): 1223-1229.

Cristofanilli M, Budd GT, Ellis MJ, Stopeck A, Matera J, Miller MC, Reuben JM, Doyle GV, Allard WJ, Terstappen L, Hayes DF (2004) Circulating tumor cells, disease progression, and survival in metastatic breast cancer. N Engl J Med 351(8): 781-791.

Cristofanilli M, Hayes DF, Budd GT, Ellis MJ, Stopeck A, Reuben JM, Doyle GV, Matera J, Allard WJ, Miller MC, Fritsche HA, Hortobagyi GN, Terstappen L (2005) Circulating tumor cells: a novel prognostic factor for newly diagnosed metastatic breast cancer. J Clin Oncol 23(7): $1420-1430$.

Danila DC, Heller G, Gignac GA, Gonzalez-Espinoza R, Anand A, Tanaka E, Lilja H, Schwartz L, Larson S, Fleisher M, Scher HI (2007) Circulating tumor cell number and prognosis in progressive castration-resistant prostate cancer. Clin Cancer Res 13(23): 7053-7058.

de Bono JS, Scher HI, Montgomery RB, Parker C, Miller MC, Tissing H, Doyle GV, Terstappen LW, Pienta KJ, Raghavan D (2008) Circulating tumor cells predict survival benefit from treatment in metastatic castration-resistant prostate cancer. Clin Cancer Res 14(19): 6302-6309.

Eisenhauer EA, Therasse P, Bogaerts J, Schwartz LH, Sargent D, Ford R, Dancey J, Arbuck S, Gwyther S, Mooney M, Rubinstein L, Shankar L, Dodd L, Kaplan R, Lacombe D, Verweij J (2009) New response evaluation criteria in solid tumours: revised RECIST guideline (version 1.1). Eur $J$ Cancer 45(2): 228-247.

Gao J, Lu M, Yu JW, Li YY, Shen L (2011) Thymidine phosphorylase/betatubulin III expressions predict the response in Chinese advanced gastric cancer patients receiving first-line capecitabine plus paclitaxel. $B M C$ Cancer 11: 177.

Haber DA, Velculescu VE (2014) Blood-based analyses of cancer: circulating tumor cells and circulating tumor DNA. Cancer Discov 4(6): 650-661.

Hartgrink HH, Jansen EPM, van Grieken NCT, van de Velde CJH (2009) Gastric cancer. Lancet 374(9688): 477-490.

Hiltermann TJ, Pore MM, van den Berg A, Timens W, Boezen HM, Liesker JJ, Schouwink JH, Wijnands WJ, Kerner GS, Kruyt FA, Tissing H, Tibbe AG, Terstappen LW, Groen HJ (2012) Circulating tumor cells in small-cell lung cancer: a predictive and prognostic factor. Ann Oncol 23(11): 2937-2942.

Hou JM, Greystoke A, Lancashire L, Cummings J, Ward T, Board R, Amir E, Hughes S, Krebs M, Hughes A, Ranson M, Lorigan P, Dive C, Blackhall FH (2009) Evaluation of circulating tumor cells and serological cell death biomarkers in small cell lung cancer patients undergoing chemotherapy. Am J Pathol 175(2): 808-816.

Hou JM, Krebs MG, Lancashire L, Sloane R, Backen A, Swain RK, Priest LJ, Greystoke A, Zhou C, Morris K, Ward T, Blackhall FH, Dive C (2012) Clinical significance and molecular characteristics of circulating tumor cells and circulating tumor microemboli in patients with small-cell lung cancer. J Clin Oncol 30(5): 525-532.

Jemal A, Bray F, Center MM, Ferlay J, Ward E, Forman D (2011) Global cancer statistics. CA Cancer J Clin 61(2): 69-90.

Krebs MG, Sloane R, Priest L, Lancashire L, Hou JM, Greystoke A, Ward TH, Ferraldeschi R, Hughes A, Clack G, Ranson M, Dive C, Blackhall FH (2011) Evaluation and prognostic significance of circulating tumor cells in patients with non-small-cell lung cancer. J Clin Oncol 29(12): $1556-1563$.

Li Y, Zhang X, Ge S, Gao J, Gong J, Lu M, Zhang Q, Cao Y, Wang DD, Lin PP, Shen L (2014) Clinical significance of phenotyping and karyotyping of circulating tumor cells in patients with advanced gastric cancer. Oncotarget 5(16): 6594-6602.

Liang H, Kim YH (2013) Identifying molecular drivers of gastric cancer through next-generation sequencing. Cancer Lett 340(2): 241-246.
Lim SHS, Becker TM, Chua W, Ng WL, de Souza P, Spring KJ (2014) Circulating tumour cells and the epithelial mesenchymal transition in colorectal cancer. J Clin Pathol 67(10): 848-853.

Lu M, Gao J, Wang XC, Shen L (2011) Expressions of thymidylate synthase, thymidine phosphorylase, class III beta-tubulin, and excision repair crosscomplementing group 1 predict response in advanced gastric cancer patients receiving capecitabine plus paclitaxel or cisplatin. Chin J Cancer Res 23(4): 288-294.

Matsusaka S, Chin K, Ogura M, Suenaga M, Shinozaki E, Mishima Y, Terui Y, Mizunuma N, Hatake K (2010) Circulating tumor cells as a surrogate marker for determining response to chemotherapy in patients with advanced gastric cancer. Cancer Sci 101(4): 1067-1071.

Miyamoto DT, Sequist LV, Lee RJ (2014) Circulating tumour cells-monitoring treatment response in prostate cancer. Nat Rev Clin Oncol 11(7): 401-412.

Mostert B, Sleijfer S, Foekens JA, Gratama JW (2009) Circulating tumor cells (CTCs): detection methods and their clinical relevance in breast cancer. Cancer Treat Rev 35(5): 463-474.

Rahbari NN, Aigner M, Thorlund K, Mollberg N, Motschall E, Jensen K, Diener MK, Buchler MW, Koch M, Weitz J (2010) Meta-analysis shows that detection of circulating tumor cells indicates poor prognosis in patients with colorectal cancer. Gastroenterology 138(5): 1714-1720.

Scartozzi M, Bittoni A, Pistelli M, Galizia E, Berardi R, Giampieri R, Faloppi L, Cascinu S (2009) Toward molecularly selected chemotherapy for advanced gastric cancer: state of the art and future perspectives. Cancer Treat Rev 35(5): 451-462.

Scher HI, Jia X, de Bono JS, Fleisher M, Pienta KJ, Raghavan D, Heller G (2009) Circulating tumour cells as prognostic markers in progressive, castration-resistant prostate cancer: a reanalysis of IMMC38 trial data. Lancet Oncol 10(3): 233-239.

Shen L, Shan YS, Hu HM, Price TJ, Sirohi B, Yeh KH, Yang YH, Sano T, Yang HK, Zhang X, Park SR, Fujii M, Kang YK, Chen LT (2013) Management of gastric cancer in Asia: resource-stratified guidelines. Lancet Oncol 14(12): e535-e547.

Tang L, Zhao S, Liu W, Parchim NF, Huang J, Tang Y, Gan P, Zhong M (2013) Diagnostic accuracy of circulating tumor cells detection in gastric cancer: systematic review and meta-analysis. BMC Cancer 13: 314 .

Theodoropoulos PA, Polioudaki H, Agelaki S, Kallergi G, Saridaki Z, Mavroudis D, Georgoulias V (2010) Circulating tumor cells with a putative stem cell phenotype in peripheral blood of patients with breast cancer. Cancer Lett 288(1): 99-106.

Tsujiura M, Ichikawa D, Konishi H, Komatsu S, Shiozaki A, Otsuji E (2014) Liquid biopsy of gastric cancer patients: circulating tumor cells and cellfree nucleic acids. World J Gastroenterol 20(12): 3265-3286.

Wang J, Wang K, Xu J, Huang J, Zhang T (2013) Prognostic significance of circulating tumor cells in non-small-cell lung cancer patients: a metaanalysis. PLoS One 8(11): e78070.

Wang M, Li Y, Gao J, Li Y, Zhou J, Gu L, Shen L, Deng D (2014) p16 Methylation is associated with chemosensitivity to fluorouracil in patients with advanced gastric cancer. Med Oncol 31(6): 988.

Youden WJ (1950) Index for rating diagnostic tests. Cancer 3(1): 32-35.

Yu M, Stott S, Toner M, Maheswaran S, Haber DA (2011) Circulating tumor cells: approaches to isolation and characterization. J Cell Biol 192(3): 373-382.

This work is published under the standard license to publish agreement. After 12 months the work will become freely available and the license terms will switch to a Creative Commons AttributionNonCommercial-Share Alike 4.0 Unported License.

Supplementary Information accompanies this paper on British Journal of Cancer website (http://www.nature.com/bjc) 